\title{
STEPWISE RELATIONSHIP OF EXERCISE FREQUENCY WITH SERUM BDNF LEVEL AMONG ADOLESCENTS
}

'Mahmoud A. Alomari, PhD and 2O.F. Khabour, PhD

'Department of Physical Education, Qatar University, Doha, Qatar and 2Department of Medical Laboratory, Jordan University of Science and Technology, Irbid, Jordan
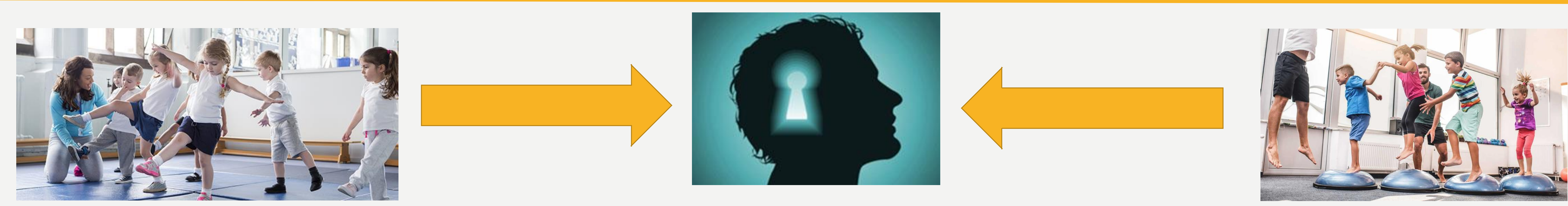

\section{INTRODUCTION}

Physical activity (PA) in adolescents is associated with numerous health benefits, including improved functions of the CV, metabolic, muscular, respiratory, immune, and neurohormonal systems (Dobbins, 12; Strong 06).

The American College of Sports Sciences recommend 3-5 times/week of moderated/vigorous exercise to improve health and fitness (Acsm, II).

Brain derived neurotrophic factor (BDNF) is essential for neural growth and functions, especially the ones related to cognitivty adolescents (Verburgh, 14; Jeon, 15). Exercise seems to enhance cognitive function (Verburgh, 14), and serum BDNF levels (Jeon, 15) in adolescents.

\section{GAPS AND PURPOSE}

However, the relationship of PA frequency with serum BDNF level is still scars in adolescents.

Examine the relationship of the frequency of participation in running exercise with serum BDNF level among adolescents.

Design and Participants

\section{METHODOLOGY}

Adolescents from $7^{\text {th }}-12^{\text {th }}$ grades were recruited to participate in the study.

Informed consents and assents were obtained from all adolescents after detailed orientation.

A self-reporting questionnaire was used to asses weekly frequency of participating in running PA.

BDNF and Physical Activity Measurements

Blood samples were drawn using venipuncture from antecubital veins into plain glass tubes while participants were sitting.

After collection, samples were spun for 8-10 minutes at $1500 \times g$ to obtain serum for BDNF measures.

Serum samples were then divided into several aliquots and immediately stored at $\neg-80^{\circ} \mathrm{C}$ for future use.

Serum BDNF was determined by ELISA.

Weekly frequency of running was self-reporting using the "SALSA" questionnaire to determine moderate/vigorous exercise.

\section{RESULTS}

\begin{tabular}{|c|c|}
\hline \multicolumn{2}{|c|}{ Adolescent characteristics ( $n=703)$} \\
\hline Variables & Mean \pm StD \\
\hline Gender (M:F) & $48.5: 51: 8$ \\
\hline Age (years) & $14.6 \pm 1.1$ \\
\hline Height $(\mathrm{cm})$ & $160.5 \pm 10.3$ \\
\hline Weight (kg) & $56.2 \pm 13.7$ \\
\hline Fat (\%) & $19.0 \pm 8.3$ \\
\hline BMI $\left(\mathbf{k g} / \mathbf{m}^{2}\right)$ & $21.9 \pm 5.3$ \\
\hline
\end{tabular}

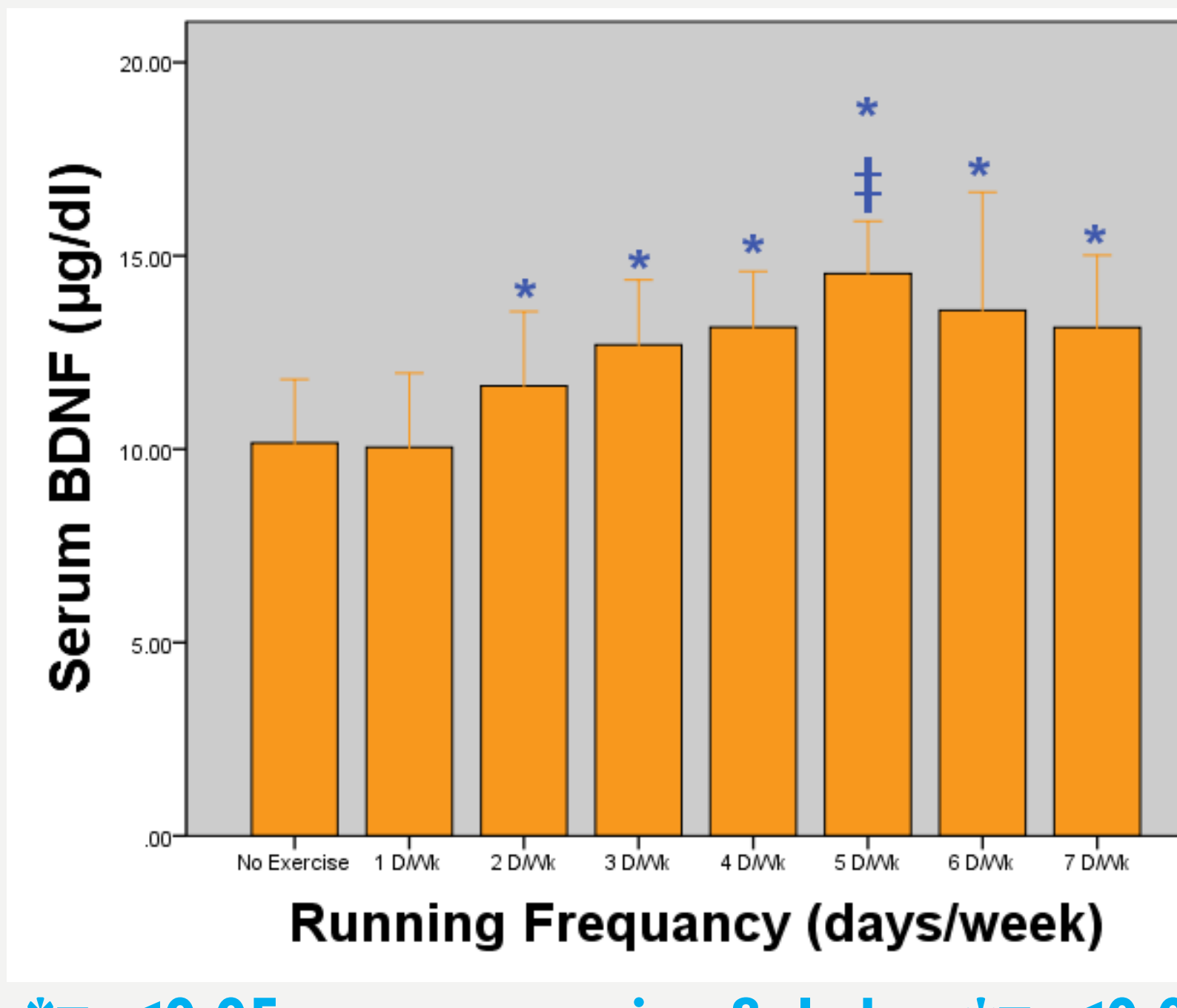

$*=0<0.05$ vs. no exercise \& I day; $\$=p<0.05$ vs. 2 days

The ANOVA revealed differences in BDNF levels according to frequency of participation in vigorous PA.

Subsequent post-hoc comparison showed that BDNF levels increased in a stepwise manner and peaked at 5 day participation in running.
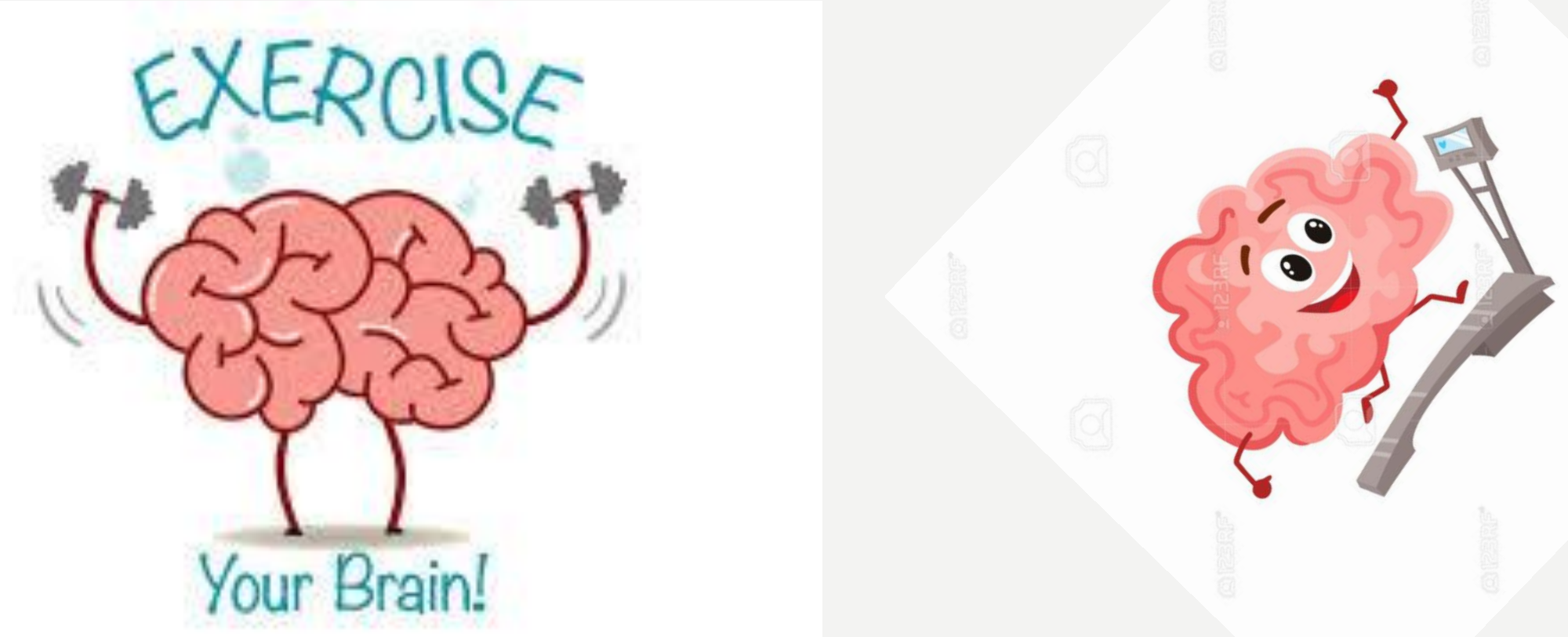

\section{CONCLUSIONS}

The study confirms the importance of PA for BDNF.

Regular participation in exercise seems to increase BDNF mRNA transcription, formation, and release in several brain compartments (Vaynman, 04). This increase is associated with promoting growth and survival of neurons, synaptic plasticity, efficacy and modulation (Seifert, 10). Consequently, cognitive function, particularly learning and memory, are improved (Joundi, I2).

In the current study, serum BDNF level increased in a stepwise fashion as frequency of exercise increased.

This is consistent with the "dose-response" principle of exercise training that indicates improvements are greater with more stimulus (i.e. exercise).

The results suggest that some exercise (3-4 d/wk) is beneficial for BDNF, however more can be even better.

Adolescents should regularly participate in exercise according to the international exercise recommendations (AcsM, 09).
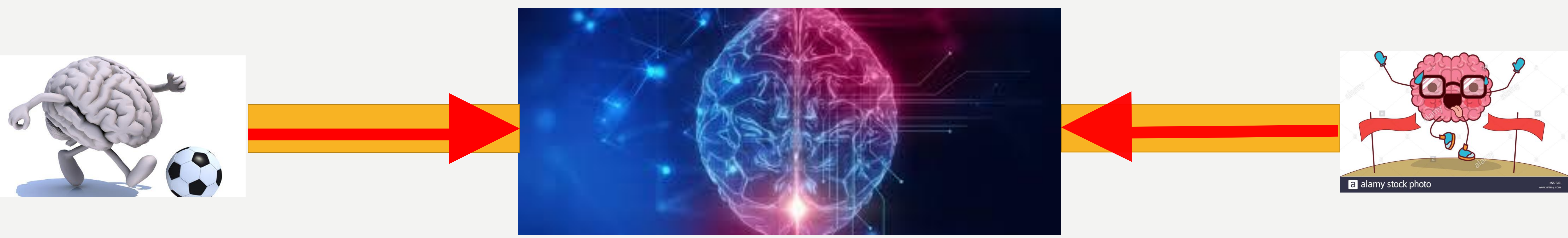

RECOMMENDATIONS

Interventional and longitudinal studies are needed to examine the long-term effects of various frequencies of exercise on serum BDNF. Additionally, studies are needed to investigate the dose-effect of exercise-induced increase in BDNF with cognitive function among adolescents.

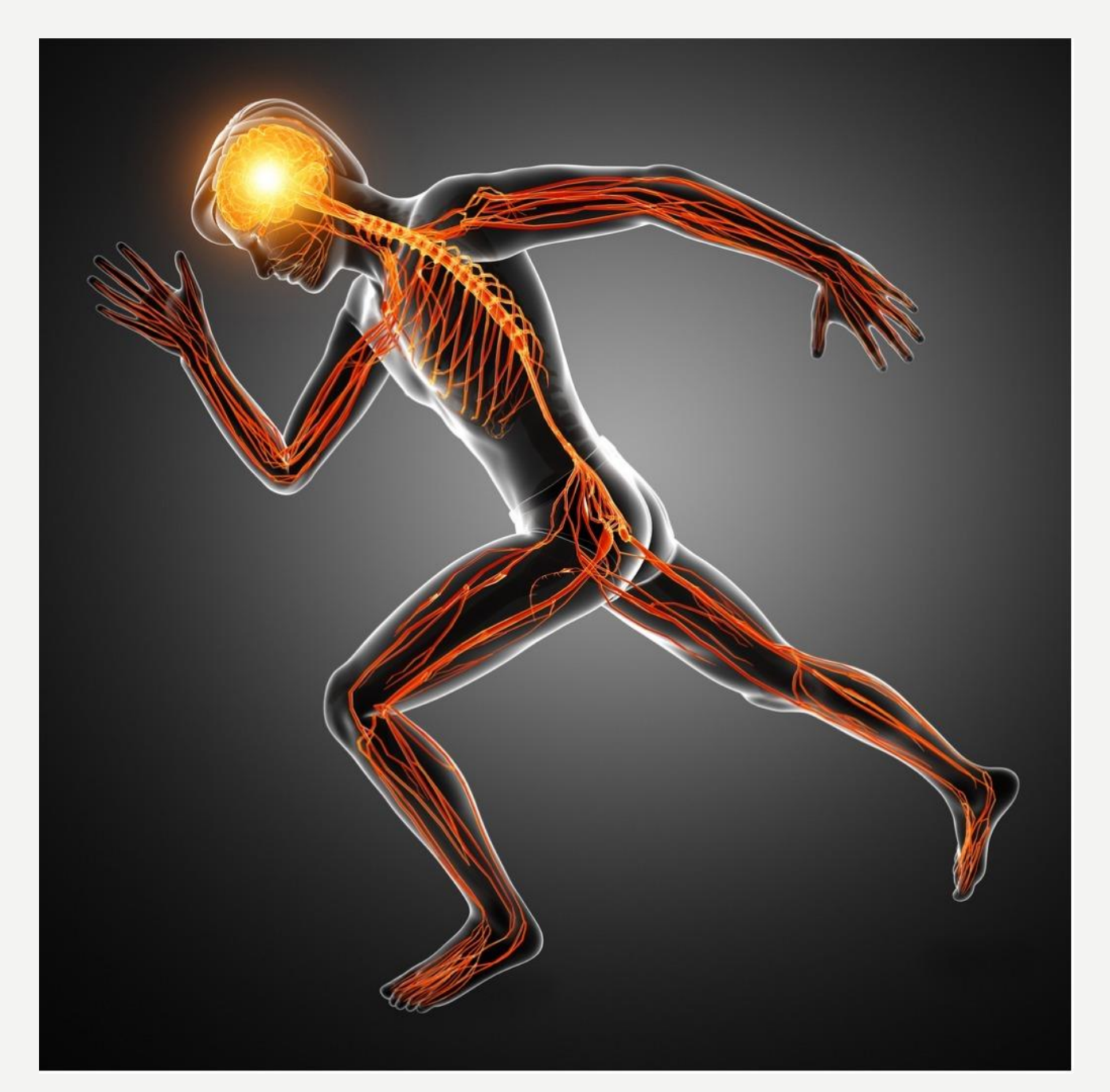

